\title{
Retrograde venous invasion in renal cell carcinoma: a complication of sinus vein and main renal vein invasion
}

\author{
Stephen M Bonsib and Ami Bhalodia \\ Department of Pathology, Louisiana State University Health Sciences Center, Shreveport, LA, USA
}

\begin{abstract}
Renal cell carcinoma, especially clear cell, gains access to the venous system as the initial route of extrarenal spread. Intravenous growth can involve extrarenal veins or renal veins in other portions of the kidney, referred to herein as retrograde venous invasion. This study investigates the incidence and defines the pathological features of retrograde venous invasion. Retrograde venous invasion is defined as rounded nodules of tumor separated from the primary tumor and in a location that conforms to the venous outflow. Nine cases of retrograde venous invasion were identified in a series of 115 renal cell carcinomas (8\%). Two blocks from each case were stained with elastic van Gieson, Masson trichrome, CD31 and desmin to evaluate intravenous involvement. All cases were staged using the 2010 TNM staging schema. The tumors ranged in size from 4.2 to $17 \mathrm{~cm}$. All cases showed sinus vein and main renal vein invasion (pT3a); three cases involved the vena cava (pT3b). Direct continuity between the primary tumor and tumor in the main renal vein was grossly evident in every case. Involved sinus veins could be followed retrograde to the cortex between renal pyramids with tumor nodules arrayed along the pyramid-cortex interface. Histologically, the involved parenchymal veins lacked a smooth muscle media and elastica. CD31 demonstrated an endothelial cell lining around many nodules. As intravenous nodules enlarged endothelium was lost, extra-venous invasion occurred and nodules coalesced and merged with the primary tumor. In conclusion, retrograde venous invasion occurred only with main renal vein involvement. Gross evaluation allowed detection in every case. Histological confirmation of intravenous nature is challenging due to the absence of smooth muscle in parenchymal veins. As retrograde growth becomes extensive nodules coalesce and merge with the primary tumor and may be included in measurement of primary tumor size if this process is unrecognized.
\end{abstract}

Modern Pathology (2011) 24, 1578-1585; doi:10.1038/modpathol.2011.124; published online 5 August 2011

Keywords: renal cell carcinoma; renal sinus; sinus veins; venous invasion

Renal cell carcinoma, especially the clear cell type, often gains access to large tumor-draining veins while the primary tumor is still intrarenal., ${ }^{1,2}$ This propensity for intravenous growth can be extensive, initially involving sinus veins, proceeding to the main renal vein, continuing into the vena cava, and on occasion, reaching the right ventricle.$^{3-5}$ This impressive tendency for intravenous growth can also involve the renal venous system in regions of the kidney distant from the primary tumor. When this occurs it may not always be recognized as an

Correspondence: Dr SM Bonsib, MD, Department of Pathology, LSU Health Sciences Center, 1541 Kings Highway, Shreveport, LA 71130-3932, USA.

E-mail: sbonsi@lsuhsc.edu

Received 7 April 2011; revised 14 June 2011; accepted 14 June 2011; published online 5 August 2011 intravenous process. One author (SB) first recognized this phenomenon several years ago. It is briefly mentioned in two recent publications but has not been reported in detail., ${ }^{6,7}$ This study investigates the incidence and defines the gross and histological features of retrograde venous invasion.

\section{Materials and methods}

In all, 115 renal cell carcinomas accessioned from 2007 to 2011 at Louisiana State University Health Sciences Center Department of Pathology were reviewed. The tumors were handled in the gross pathology laboratory by one or both of the authors, and were opened through the venous system as previously described. ${ }^{6}$ All cases were staged using the 2010 TNM staging schema. ${ }^{8}$ Cases with retrograde 
cortical venous invasion were identified as part of an ongoing study of invasive pathways for renal cell carcinomas. This project received Institutional Review Board approval.

Main renal vein invasion was defined as tumor in an enlarged vein visible at the renal hilum before opening the specimen. Retrograde venous invasion was defined as rounded or elongated nodules of tumor separated from the primary tumor by uninvolved renal parenchyma. Tumor nodules must be in a location that conforms to the normal venous outflow of the kidney, that is, between renal pyramids or at the cortical-medullary junction.

Two blocks of tissue from each case of retrograde venous invasion were stained with a short panel of histochemical and immunohistochemical stains in an attempt to demonstrate the intravascular nature of the tumor nodules. These stains included elastic van Gieson stain, Masson trichrome stain, desmin and CD31. Stained sections were reviewed for evidence of vein wall and endothelial cell lining.

\section{Results}

A total of 115 renal cell carcinomas were reviewed consisting of 72 cases of clear-cell renal cell carcinoma, 24 cases of papillary renal cell carcinoma, five cases of chromophobe renal cell carcinoma, six cases of renal cell carcinoma, unclassified and eight other types of renal cell carcinoma. Nine cases, seven clear cell carcinomas and two renal cell carcinomas, unclassified, demonstrated retrograde venous invasion representing $8 \%$ of all renal cell carcinomas and $22 \%$ of pT3 tumors (Table 1 ). All tumors with retrograde venous invasion showed involvement of renal sinus veins and the main renal vein. Three of nine tumors also extended into the vena cava (Table 2). This included two of three rightsided tumors but only one of six left-sided tumors. Tumor sizes ranged from 4.2 to $17 \mathrm{~cm}$ (Table 2). Tumors were aggressive in five patients with metastatic disease at time of nephrectomy to lung (three cases), lymph nodes (three cases), bone (one case) and adrenal (one case). Two of the four remaining cases had no followup and the remaining two cases had only a short followup of 1 and 4.5 months, respectively.

Table 1 Renal cell carcinoma: type, stage and incidence of retrograde venous invasion

\begin{tabular}{lrrrrrr}
\hline Type & \#Cases & $\# p T 1$ & \#pT2 & \#pT3 & $\begin{array}{c}\text { \#Retro- } \\
\text { grade }\end{array}$ & $\begin{array}{c}\text { (\%) Retro- } \\
\text { grade }\end{array}$ \\
\hline Clear cell & 72 & 33 & 6 & 33 & 7 & $10 \%$ \\
Papillary & 24 & 18 & 1 & 5 & 0 & $0 \%$ \\
Chromophobe & 5 & 2 & 3 & 0 & 0 & $0 \%$ \\
Unclassified & 6 & 3 & 1 & 2 & 2 & $33 \%$ \\
Other & 8 & 6 & 1 & 1 & 0 & $0 \%$ \\
Total & 115 & 62 & 12 & 41 & 9 & $8 \%$ \\
\hline
\end{tabular}

In all cases with retrograde venous invasion the unopened nephrectomy specimen had grossly obvious main renal vein involvement. By opening the specimens through the venous system, contiguous venous involvement from the main renal vein to the primary tumor was evident (Figures 1 and 2). In addition, contiguous venous involvement from the main renal vein to sinus veins, and proximal, to veins exiting the cortex from non-tumorous areas was readily visible in 7/9 cases (Figures 1 and 2). In two cases additional parallel sections were required to clearly demonstrate the process (Figure 3).

In all cases multiple sinus veins were involved. Retrograde venous invasion was most often observed in renal lobes adjacent to the primary tumor, but was also in observed distant poles in several cases (Figure 4a). When retrograde venous invasion was extensive the intravenous tumor nodules involved the entire kidney (Figure 5). In all cases rounded or elongated $0.1-1.0 \mathrm{~cm}$ sized nodules were visible between renal pyramids or arrayed along the

Table 2 Pathological features of renal cell carcinomas with retrograde venous spread

\begin{tabular}{lcccc}
\hline Carcinoma type & Size & Grade & Main renal vein & Vena cava \\
\hline Clear cell \#1 & 6.5 & 3 & Pos & Pos \\
Clear cell \#2 & 8.4 & 4 & Pos & Pos \\
Unclassified \#1 & 17 & 3 & Pos & Pos \\
Clear cell \#3 & 4.2 & 2 & Pos & Neg \\
Clear cell \#4 & 5 & 2 & Pos & Neg \\
Clear cell \#5 & 7 & 3 & Pos & Neg \\
Clear cell \#6 & 7.5 & 2 & Pos & Neg \\
Unclassified \#2 & 12 & 4 & Pos & Neg \\
Clear cell \#7 & 12.5 & 2 & Pos & Neg \\
\hline
\end{tabular}

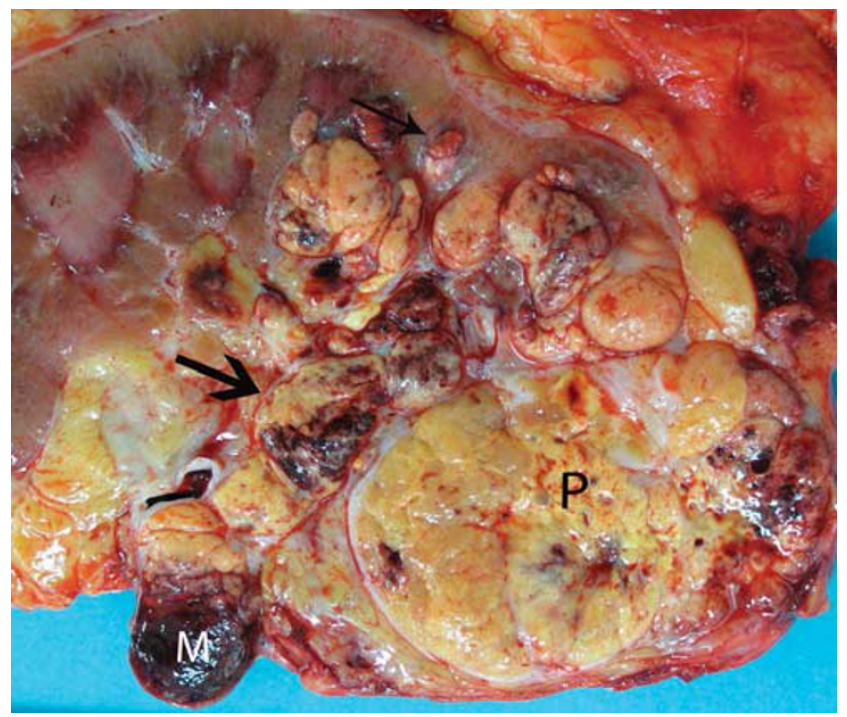

Figure 1 This clear-cell carcinoma invaded the inferior vena cava, the main renal vein $(\mathrm{M})$ and sinus veins (large arrow). Nodules of retrograde venous invasion tumor are also present within the renal parenchyma (small arrow). P, primary tumor. 

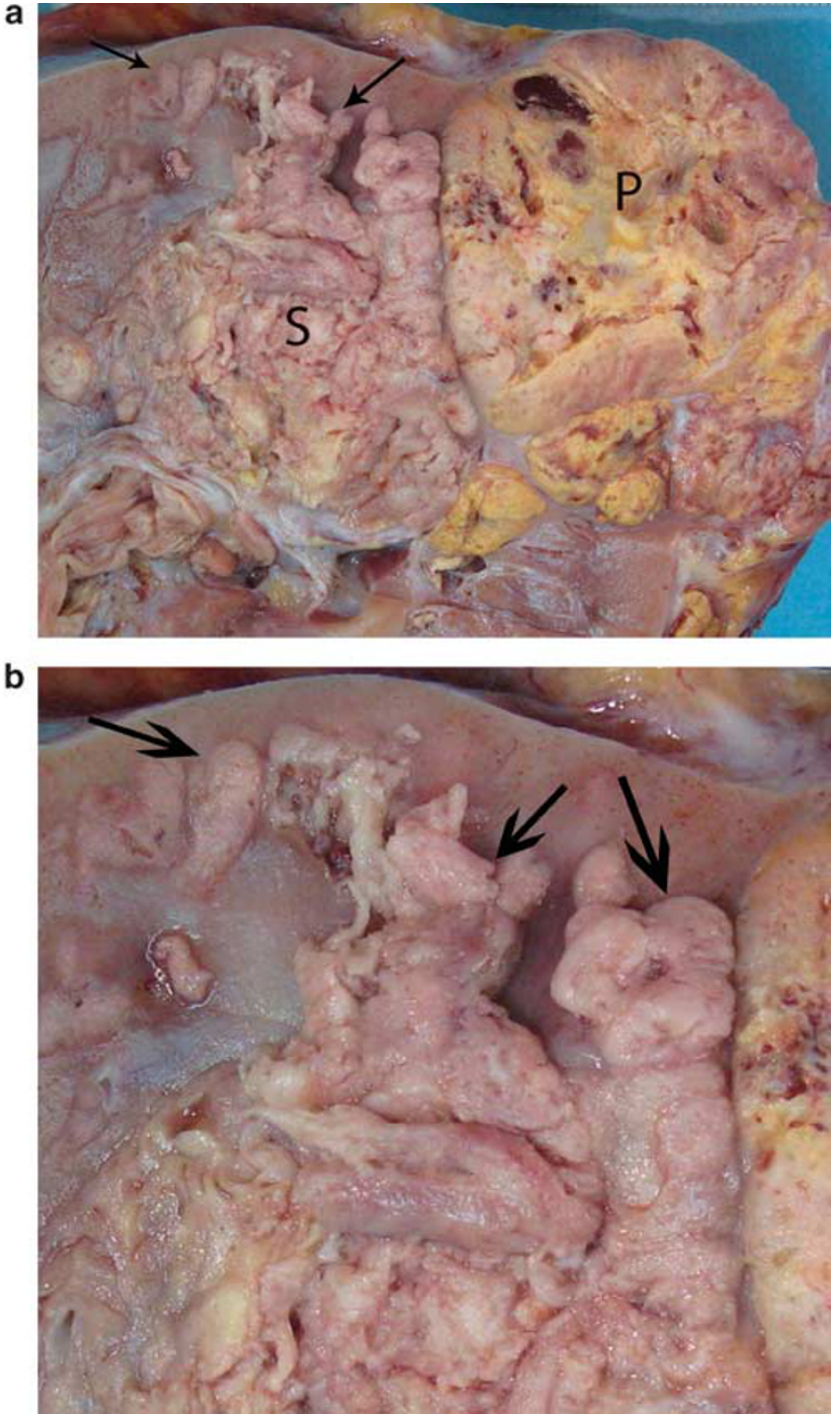

Figure 2 (a) This clear-cell carcinoma shows a partially necrotic primary tumor $(\mathrm{P})$ and confluent sinus invasion (S). Adjacent to the primary tumor, tumor invades intralobar veins and multiple arcuate veins (arrows). (b) There are thick cylinders of tumor between the renal pyramids and arcades of tumor arrayed along the corticomedullary junction (arrows). All tumor nodules represent intravascular tumor.

cortical-medullary junction representing intralobar and arcuate vein involvement, respectively (Figures 2b, 3 and 5). In addition, some cases showed cortical nodules consistent with interlobular vein involvement. As the intravenous nodules closest to the primary tumor enlarged they became confluent, coalescing into a multinodular mass making discrimination of the limits of the primary tumor from adjacent retrograde venous invasion challenging (Figure 5). In some cases the intravenous tumor was a different color than the primary tumor, or was viable while the primary tumor was necrotic aiding gross recognition (Figures 2 and 3 ).

Microscopically, retrograde venous invasion was recognized by the combination of location, relationships and evidence of pre-existing vein. Location

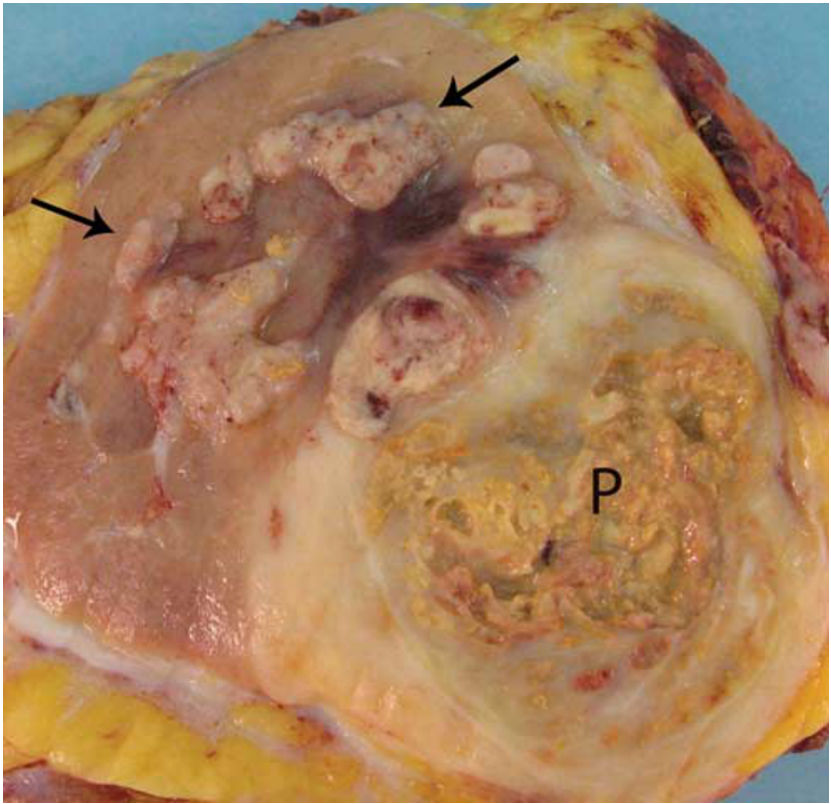

Figure 3 This clear-cell renal cell carcinoma showed extensive sinus vein invasion on the initial section. This additional parallel section shows obvious retrograde venous invasion (arrows) with arcade of nodules draped around renal pyramids. Notice that the primary tumor is largely necrotic while the intravenous tumor appears viable. $\mathrm{P}$, primary tumor.

refers to the presence of tumor nodules between renal pyramids or bracketed by renal medulla and cortex, the normal location of intralobar and arcuate veins (Figures 4b, 6 and 7). Tumor extending from arcuate veins into one or more interlobular vein tributaries, was frequently identified (Figure 8). Relationship refers to the frequent presence of an artery adjacent to rounded tumor nodules, pertinent because renal arteries travel parallel to renal veins (Figures 6 and 7). When tumor nodules were small involving arcuate and intralobular veins, there was often a zone of separation between tumor and the vein wall, an obvious endothelial cell lining visible (Figures 4b, 8 and 9).

Identification of a pre-existing vein structure was impossible when an endothelial-lined zone of separation between tumor and vein wall was absent, as renal parenchymal veins lack appreciable smooth muscle media or elastica. ${ }^{1}$ This was affirmed with Masson trichrome and elastic stains and with staining for desmin. When retrograde venous invasion involved intralobar veins between the renal pyramids, a short interrupted segment of apparent vein wall smooth muscle media could be demonstrated by trichrome and desmin stains in some cases. However, when tumor involved arcuate veins and cortical interlobular veins, no vein wall smooth muscle media was ever apparent (Figure 10). Similarly, elastic stains failed to highlight a venous structure in any sized vein (Figure 11).

Trichrome and elastic stain demonstrated extensive interstitial fibrosis adjacent to the tumor nodules and extending some distance throughout 

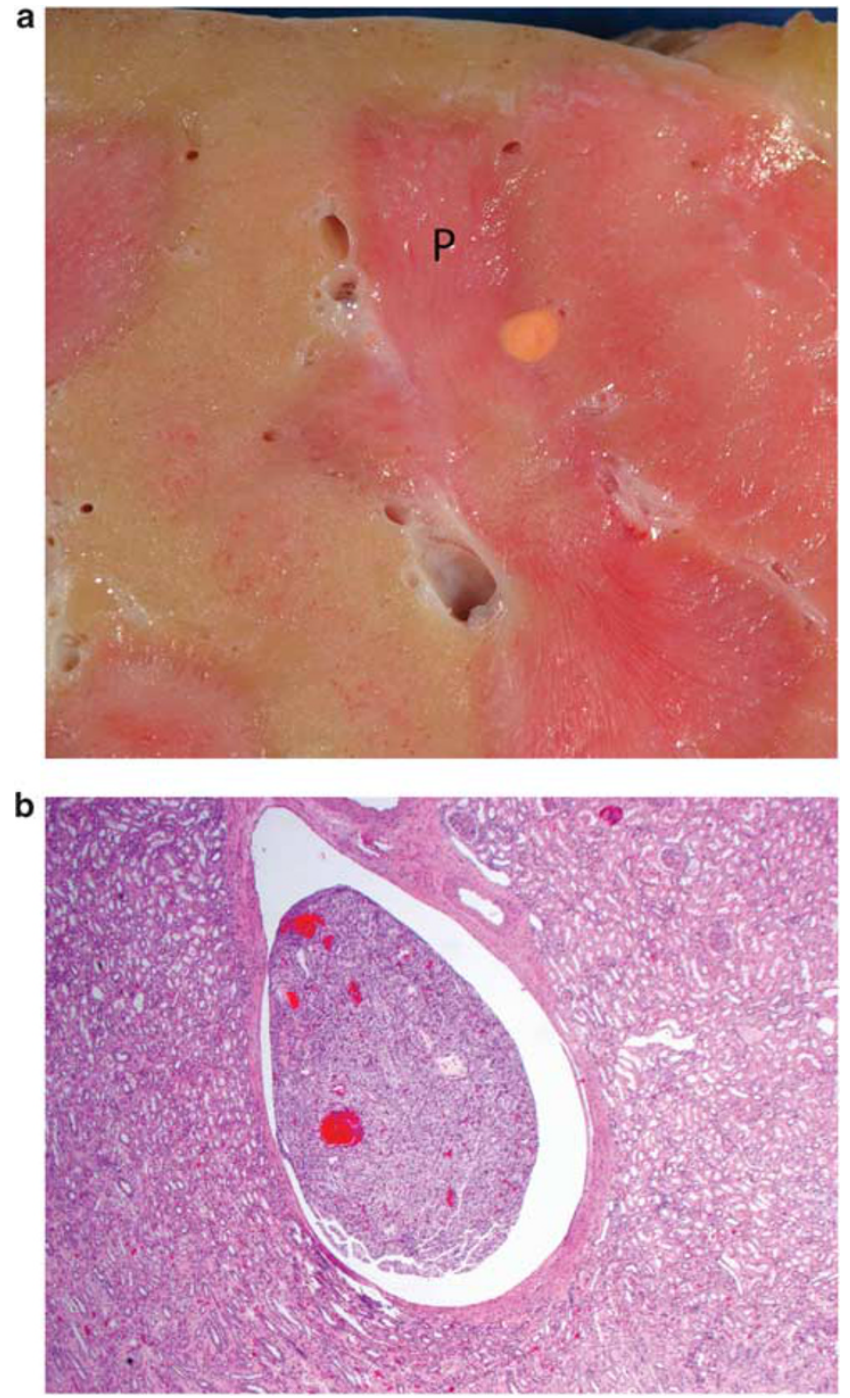

Figure 4 (a) This arcuate vein contains tumor. It was located in a pole opposite the primary tumor. Grossly, it could be mistaken for a second primary tumor. P, renal pyramid. (b) This section from the tumor in panel a confirms that it is an arcuate vein located at the corticomedullary junction.

the cortex (Figure 11). This tubulointerstitial scarring is likely the result of chronic venous outflow obstruction. Desmin and trichrome stains allowed demonstration of feeder vessels, presumably recruited from pre-existing parenchymal vessels. These vessels entered the intravenous tumor at numerous points along its course as previously described. ${ }^{1}$

CD31 was the most useful stain in demonstration of the intravenous nature of tumor nodules. CD31 often showed a complete or partial retention of an endothelial cell lining surrounding the intravenous tumor (Figures 12a and b). In cases of clear cell carcinoma, CD31 highlighted a delicate capillary lattice within the intravenous tumor, definitional of clear cell carcinoma by World Health Organization criteria. ${ }^{9}$ As nodules of retrograde venous invasion progressively enlarged, tumor becomes apposed to the vein wall obliterating the endothelial cell lining.

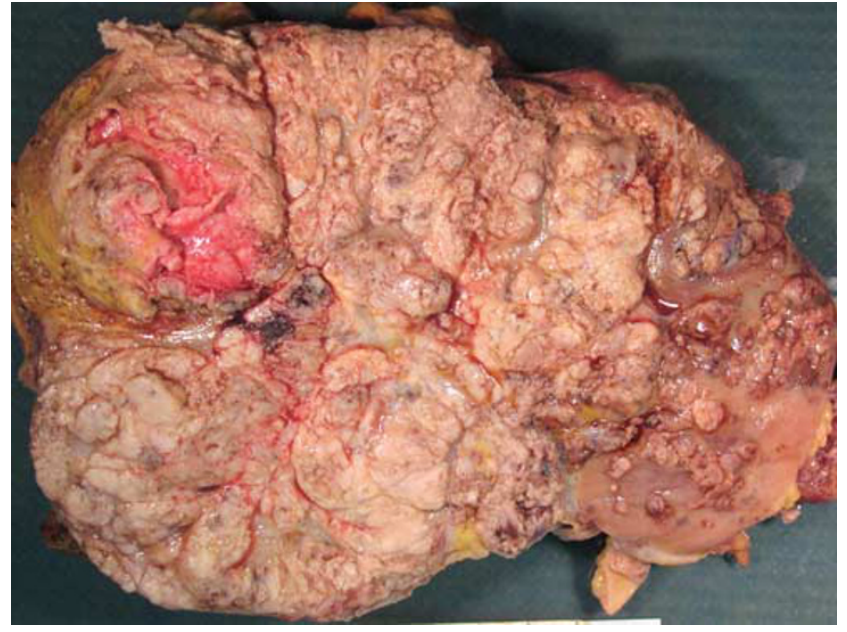

Figure 5 This clear-cell renal cell carcinoma shows the primary tumor on the left and florid retrograde venous invasion on the right. The confluence of rounded nodules in between represents extensive intravenous tumor that makes determination of the limits of the primary tumor essentially impossible.

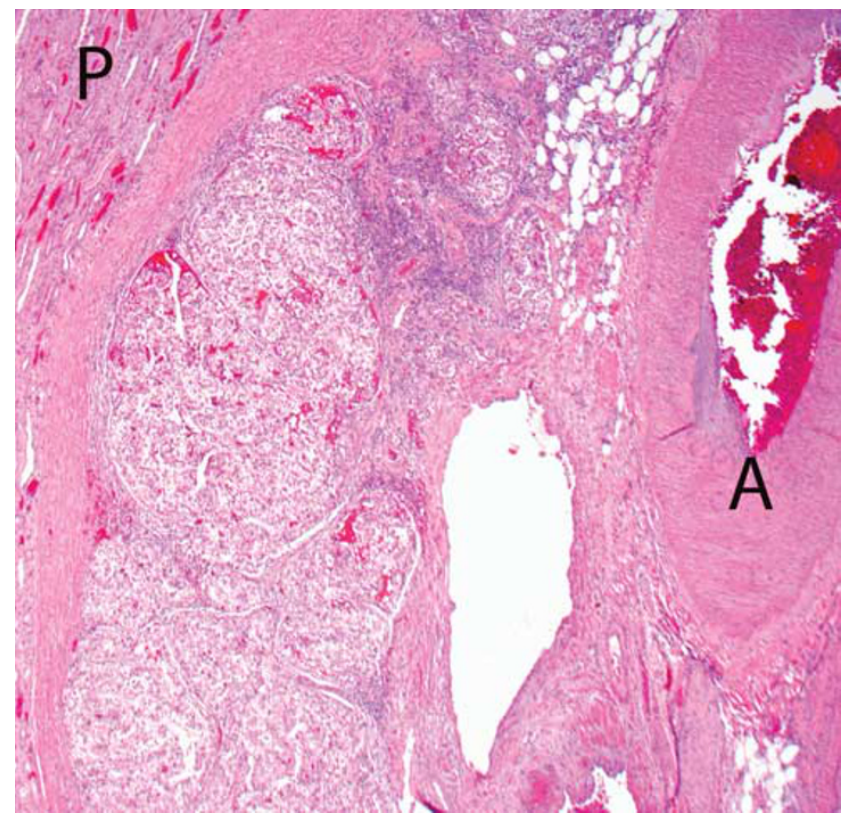

Figure 6 This shows intravenous tumor extending from sinus veins toward the parenchyma between renal pyramids $(\mathrm{P})$. The accompanying artery (A) is present.

Tumor could then be observed extending into the renal parenchyma in sites lacking a CD31-positive endothelial lining (Figure 13).

The intravenous tumor regardless of the degree of venous enlargement or the caliber of vein involved, from the main renal vein to interlobular veins, invariably contained largely viable tumor. Focal necrosis was observed in a single case of renal cell carcinoma, unclassified (Figure 8). Significant thrombosis was not present in any case. When thrombus was present it was limited to a thin layer at the edge or at the leading front of intravenous 


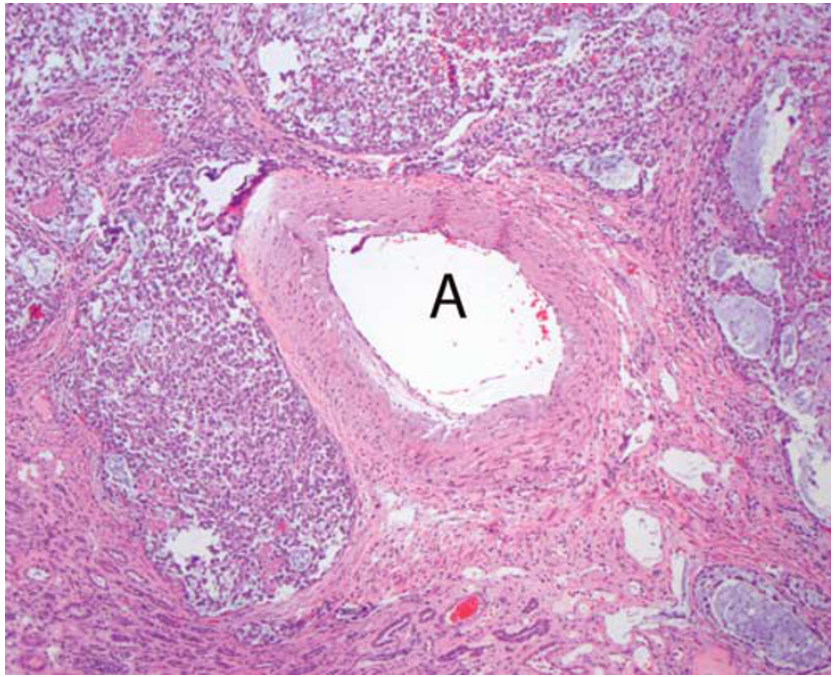

Figure 7 This renal cell carcinoma, unclassified shows intravascular tumor within a cluster of arcuate veins with a central arcuate artery (A).

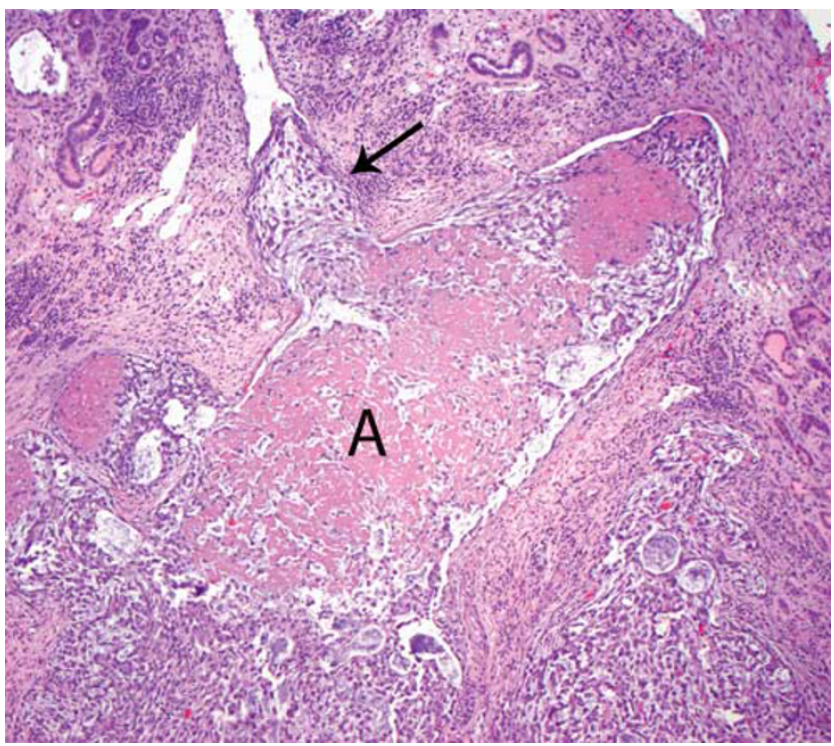

Figure 8 This dilated arcuate vein (A) contains focally necrotic renal cell carcinoma, unclassified that is invading into an intralobular vein (arrow).

tumor. In other words tumor thrombus, a reference commonly made to intravenous tumor, is not an accurate representation of the intravenous process. As the cortical nodules enlarged tumor invaded the interstitium and the nodules coalesced into multinodular masses. Demonstration of the intravenous origin of these confluent nodules became impossible but was inferred by examination of adjacent, as yet, not confluent intravenous nodules.

\section{Discussion}

Retrograde venous invasion has been briefly mentioned in the renal cell carcinoma literature, and

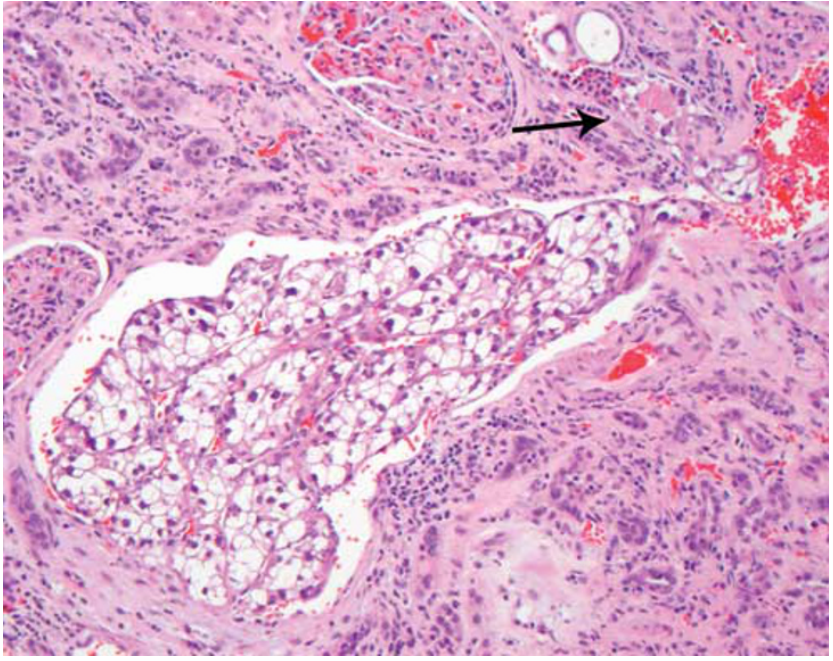

Figure 9 This is an interlobular vein filled with tumor that is branching into a small tributary vein (arrow).

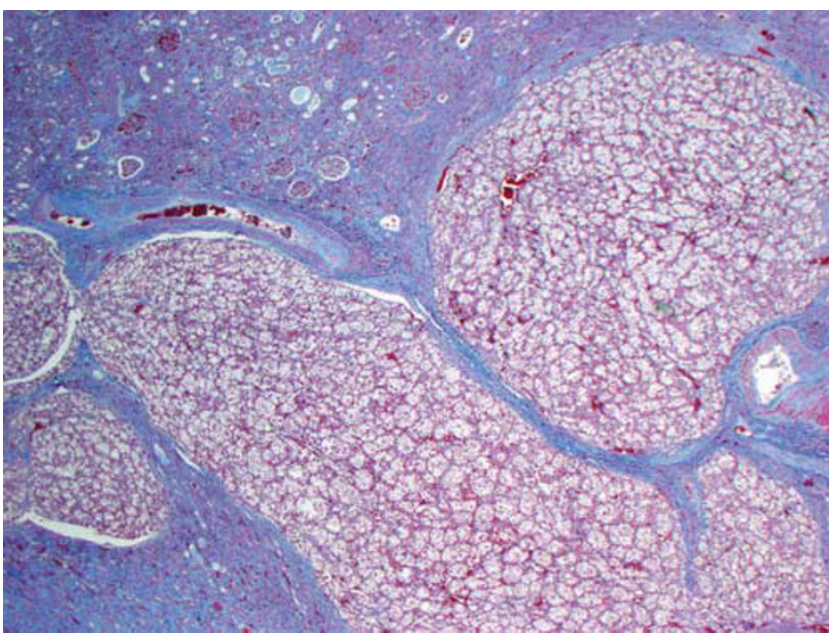

Figure 10 This collection of arcuate veins filled with tumor shows no evidence of a smooth muscle media on trichrome stain. Masson trichrome stain.

therefore, is a process that may not be widely recognized., $\mathrm{A}$ recent case report contained a beautiful gross photograph of a nephrectomy specimen that we regard as showing retrograde venous invasion. ${ }^{10}$ It was correctly interpreted as an 'infiltrative' process but its intrinsic venous nature was not mentioned. This study of retrograde venous invasion was designed to investigate this process in greater detail.

Retrograde venous involvement occurred in approximately $10 \%$ of all tumors and $22 \%$ of pT3a tumors. This process reflects aggressive behavior, as five of nine cases presented with metastatic disease. The majority of tumors were clear-cell renal cell carcinomas, not surprising in light of its remarkable diathesis for venous invasion. Although the only other category of tumor with retrograde venous invasion was renal cell carcinoma, unclassified, one author (SB) has seen 12 other cases of retrograde 


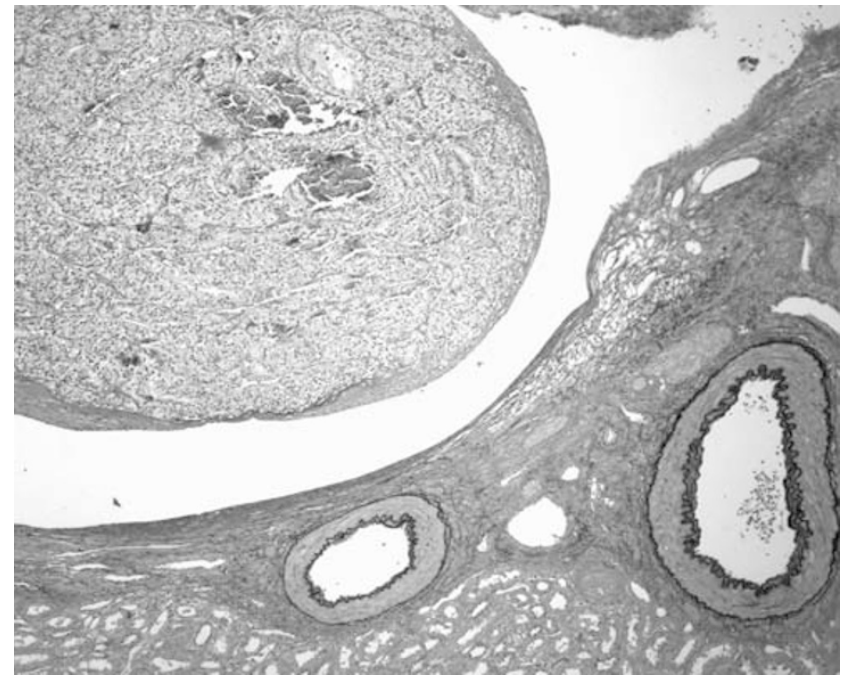

Figure 11 No elastica is visible in this intralobar vein filled with tumor. The elastica of the adjacent arteries are clearly demonstrated. van Gieson elastic stain.
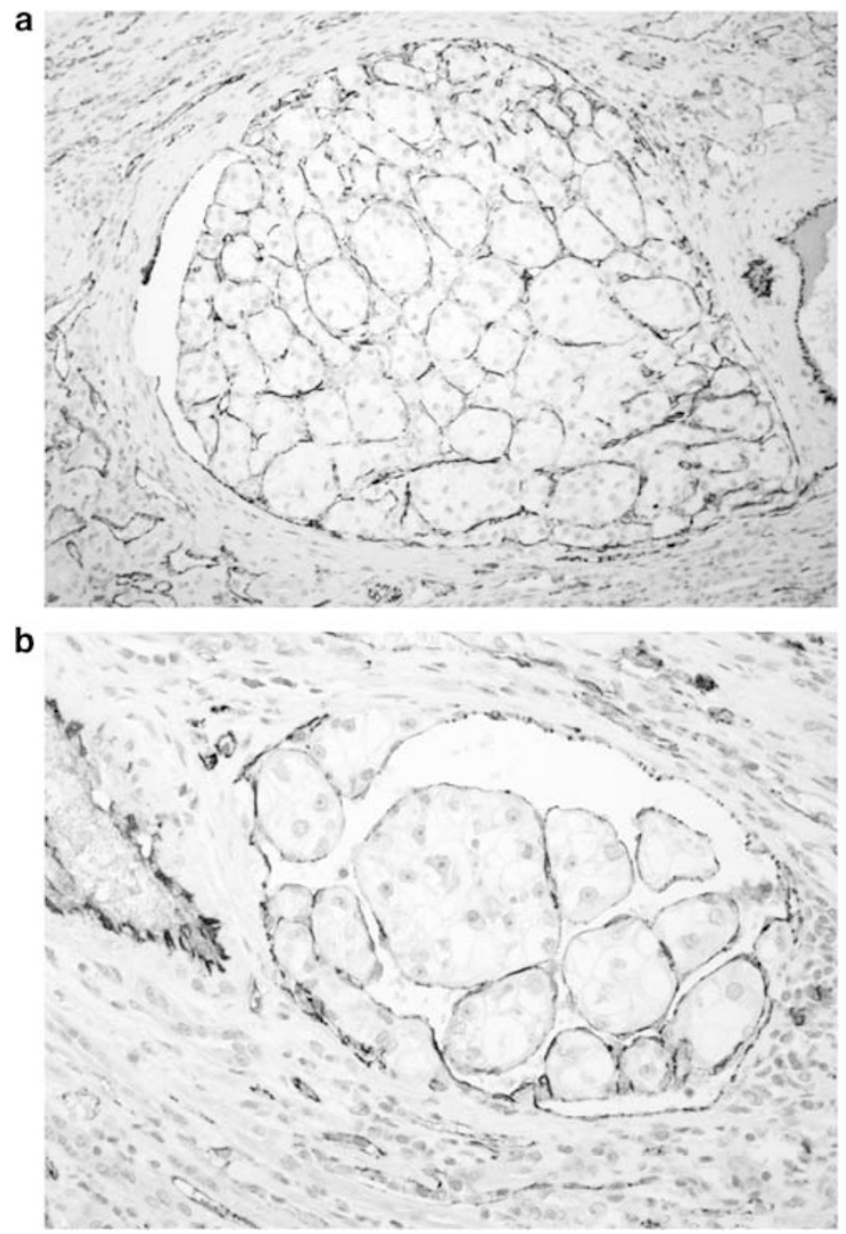

Figure 12 (a) This arcuate vein is filled with clear-cell carcinoma but shows a nearly complete endothelial cell lining and the delicate tumor capillary lattice. An artery is to the right. CD31 stain. (b) This small interlobular vein is filled with clear-cell carcinoma and shows a nearly complete endothelial cell lining and the delicate tumor capillary lattice. CD31 stain.

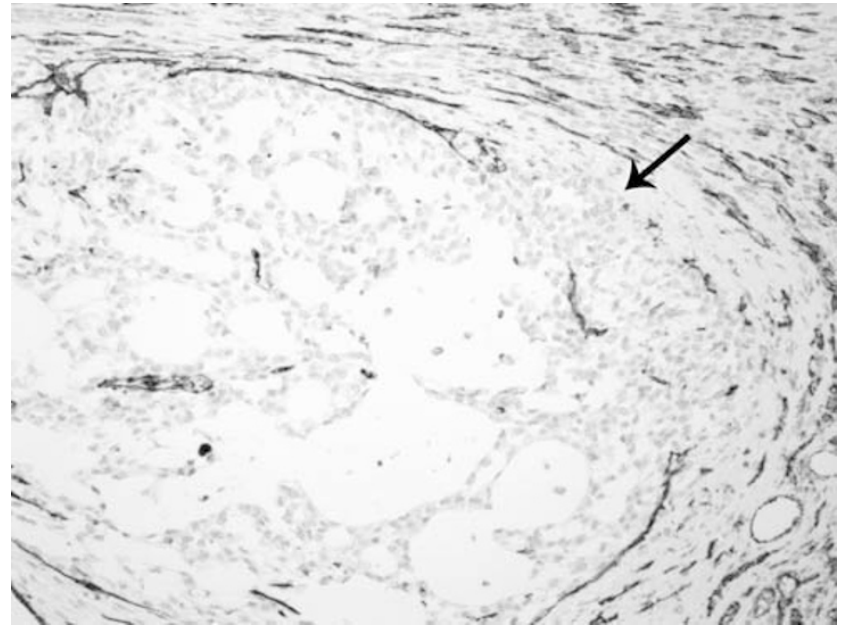

Figure 13 Tumor in this arcuate vein has begun to focally invade through the wall (arrow) obliterating the endothelial cell lining. CD31 stain.

venous invasion not included in this study, as they were not sampled specifically for this finding. This group included cases of papillary renal cell carcinoma. Presumably, this phenomenon may occur with any tumor that shows extensive renal sinus vein and main renal vein involvement. If so, any renal cell carcinoma type, even urothelial carcinoma may, on occasion, shows retrograde venous invasion.

Retrograde venous invasion was only observed when both renal sinus veins and the main renal vein were involved. This suggests that retrograde venous growth is not favored until the elongating plug of intravenous tumor has occluded the venous outflow from veins draining non-tumor regions of the kidney. Presumably tumor can then extend proximally into sinus venous tributaries that merge with veins draining the primary tumor. Growth may be favored at these sites of least resistance, as blood flow in these veins would be absent or negligible. Although to date it has not been identified, it is possible that tumor may show retrograde venous invasion without sinus vein and main renal vein involvement, as there is extensive collateral venous communication between arcuate venous systems of adjacent renal lobes. ${ }^{11,12}$

The literature on $\mathrm{pT} 3 \mathrm{a} / \mathrm{b}$ renal cell carcinoma involving renal vein and vena cava, especially the urology and radiology literature, consistently refers to intravenous tumor as tumor 'thrombus'..$^{3,4}$ This and a previous study have demonstrated that socalled tumor 'thrombus' is largely and often entirely a cylinder of solid viable tumor in direct continuity with the primary tumor. ${ }^{1}$ The word tumor thrombus is misleading and we recommend that its use be discontinued.

Identification of retrograde venous invasion is usually straightforward once familiar with this phenomena, the purpose of this report. It is easiest 
to recognize on gross examination of the bivalved specimen. Recognition is facilitated if the bivalve step is performed along the venous system but requires understanding of the normal renal venous outflow. The normal venous outflow begins with cortical interlobular veins, which empty into arcuate veins at the corticomedullary junction. These converge with other arcuate veins and exit the renal parenchyma between renal pyramids. Thus, nodules of tumor located between renal pyramids and arrayed along the corticomedullary junction in cases with main renal vein involvement likely represent retrograde venous invasion. Histological demonstration of retrograde venous invasion is challenged by the absence of smooth muscle media or elastica in the normal venous outflow of the kidney. Although the endothelial lining of involved veins can be demonstrated by CD31 stain in some affected vessels, its presence is effaced as intravenous tumor enlarges and invades the interstitium. The rounded contour and presence of an adjacent artery are useful but non-specific findings that imply an intravenous origin of the nodules.

Once affected parenchymal veins become massively dilated, tumor attaches to the vein wall obliterating the endothelial lining. Invasion through the vein wall may then occur with confluence of originally intravenous tumor nodules. This confluence makes recognition of its intravenous history difficult as such nodules merge with the primary tumor. This confluence and merger of intravenous nodules could falsely exaggerate the size of the primary tumor and may explain why many renal cell carcinoma, particularly clear-cell carcinoma, commonly have a multinodular gross appearance.

We recommend that retrograde venous invasion should be carefully considered when renal cell carcinoma involves the main renal vein. If retrograde venous invasion or sinus vein invasion is identified it should be excluded from measurement of the primary tumor size. If argued otherwise, then by extrapolation main renal vein and vena cava invasion should be included in primary tumor size measurement, an illogical concept. As retrograde venous invasion may not be widely recognized studies that correlate tumor size with outcome may have included cases of pT3 tumors in which primary tumor size was overestimated. The worsening prognosis documented with increasing tumor size may partially reflect the poor prognosis of tumors with intravenous invasion. ${ }^{13-18}$

In summary, we describe the gross and microscopical features of retrograde venous invasion by renal cell carcinoma, an invasive process that may be under-recognized. Retrograde venous invasion is most common in clear-cell renal cell carcinoma, but should be considered in any tumor type when the main renal vein is involved. Opening tumor nephrectomies through the venous system coupled with knowledge of the normal venous outflow of the kidney, allows most cases to be recognized before microscopical evaluation. In cases with extensive retrograde venous invasion intravenous tumor nodules can become large and achieve confluent growth, making accurate determination of primary tumor size difficult. If tumor size is to remain a valid prognostic and staging parameter, accurate measurements are mandatory; otherwise, large tumor size becomes a surrogate marker for invasive growth, especially in cases of clear-cell carcinoma where intravenous invasion is so common.

\section{Disclosure/conflict of interest}

The authors declare no conflict of interest.

\section{References}

1 Bonsib SM. Renal veins and venous extension in clear cell renal cell carcinoma. Mod Pathol 2007;20: 44-53.

2 Bonsib SM. The renal sinus is the principal invasive pathway: a prospective study of 100 renal cell carcinomas. Am J Surg Pathol 2004;28:1594-1600.

3 Wagner B, Patard JJ, Méjean A, et al. Prognostic value of renal vein and inferior vena cava involvement in renal cell carcinoma. Eur Urol 2009;55:452-459.

4 Katkooti D, Murugesan M, Ciancio G, et al. Tumor thrombus involving the inferior vena cava in renal malignancy: is there a difference in clinical presentation and outcome among right and left side tumors? Int Braz J Urol 2009;35:652-657.

5 Pouliot F, Shuch B, LaRochelle JC, et al. Contemporary management of renal tumors with venous tumor thrombus. J Urol 2010;184:838-841.

6 Bonsib SM. Microscopic assessment, dissection protocols and histologic sampling strategy for renal cell carcinoma. Diag Histopathol 2008;14:151-156.

7 Bonsib SM, Pei Y. The non-neoplastic kidney in tumor nephrectomy specimens: what can it show and what is important? Adv Anat Pathol 2010;17:235-250.

8 Edge SB, Byrd DR, Compton CC, et al (eds). Kidney In: AJCC Cancer Staging Handbook, 7th edn. Springer: New York, 2010, pp 547-560.

9 Grignon DJ, Eble JN, Bonsib SM, et al. Clear cell renal cell carcinoma, In: Eble JN, Sauter G, Epstein JI, Sesterhenn IS (eds). Tumors of the Urinary System and Male Genital System. IARC Press: Lyon, France, 2004, pp 23-25.

10 Hecht EM, Hindman N, Huang WC, et al. Extensive infiltrating renal cell carcinoma with minimal distortion of the renal anatomy mimicking benign renal vein thrombosis. Am J Kid Dis 2010;55:957-971.

11 Hodson CJ. The renal parenchyma and its blood supply. Curr Prob Diagn Radiol 1978;7:1-32.

12 Sykes D. The correlation between renal vasculature and the lobulation of the kidney. $\mathrm{Br} J$ Urol 1964;36:549-555.

13 Bell ET. A classification of renal tumors with observations on the frequency of the various types. J Urol 1938;39:338-243.

14 Frank I, Blute ML, Leibovich BC, et al. pT2 classification for renal cell carcinoma. Can its accuracy be improved? J Urol 2005;173:380-384. 
15 Klatte T, Patard J-J, Goel RH, et al. Prognostic impact of tumor size on pT2 renal cell carcinoma: an international multicenter experience. J Urol 2007;178:35-40.

16 Lam JS, Klatte T, Patard JJ, et al. Prognostic relevance of tumor size in T3a renal cell carcinoma: a multicenter experience. Eur Urol 2007;52:156-162.
17 Thompson RH, Hill JR, Babayev Y, et al. Metastatic renal cell carcinoma risk according to tumor size. J Urol 2009;182:41-45.

18 Nguyen MM, Gill IS. Effect of renal cancer size on the prevalence of metastases at diagnosis and mortality. J Urol 2009;181:1020-1027. 